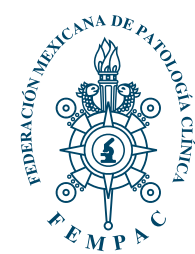

Palabras clave: Spinning, niveles

de glucosa, niveles plasmáticos de lípidos, metabolismo.

Keywords: Spinning, glucose levels, plasma lipid levels, metabolism.

* Decano de

Ciencias de la Salud,

Universidad del

Fútbol y Deporte,

Sede. Pachuca, Hgo.

* Profesor e

Investigador del

Instituto Nacional

de Aprendizaje,

Habilidades e

Investigación de

las Ciencias, A.C.

INAHIC, Zapopan, Jal.

${ }^{\S}$ Líder de Ciencias de

la Salud, Universidad

del Fútbol y Deporte.

Pachuca, Hgo.

" Secretaria Técnica de la línea de Ciencias de

la Salud, Universidad del Fútbol y Deporte,

Pachuca, Hgo.

| J Jefa de Enseñanza

e Investigación,

Centro de Excelencia

Médica en la Altura,

Universidad del

Fútbol y Deporte,

Pachuca, Hgo.

** Presidente,

Federación Mexicana

de Medicina del

Deporte, CONADE,

México, D.F.

\# Director Médico

de Laboratorio

internacional de

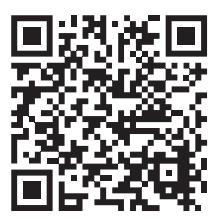

\section{Práctica de spinning y respuesta metabólica en niveles de glucosa y lípidos séricos}

\author{
Spinning practice and metabolic response \\ in blood glucose and lipid levels
}

Rivera-Cisneros Antonio Eugenio,* Sánchez-González Jorge Manuel, ${ }^{\ddagger}$ Cárdenas-González Jorge, ${ }^{\S}$ Noriega-Muro Itze," Martínez-Vega Karen," Gómez-Ballesteros Felipe Horacio,**

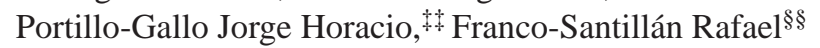

\section{RESUMEN}

La actividad de spinning es una modalidad de actividad aeróbica. Su práctica presenta diferentes coreografías, en donde la variación de la intensidad del ejercicio produce un amplio espectro de respuestas en las variables metabólicas corporales, como la glucosa. Dada su popularidad y escasa información sobre las respuestas metabólicas, se llevó a cabo el presente estudio, dirigido a valorar la respuesta de la glucemia a una clase típica de una hora de duración y niveles de lípidos. Participaron 23 voluntarios con sobrepeso (IMC $\left.>25 \mathrm{~kg} / \mathrm{m}^{2}\right)$, (11 hombres y 12 mujeres); promedio de edad de $35 \pm 7$ años, con dieta habitual estandarizada la semana previa. Los niveles hemáticos de lípidos fueron superiores a los valores de idealidad, su respuesta fue de incremento, asociado a hemoconcentración (6\%). La glucosa se elevó 20 $\mathrm{mg} / \mathrm{dL}$ y $14 \mathrm{mg} / \mathrm{dL}$ en hombres y mujeres, respectivamente. Los datos indican seguridad en el metabolismo, cuando el ejercicio es practicado con método, graduando la intensidad y los intervalos del mismo. Más investigaciones deberán caracterizar esta respuesta con otras rutinas y modalidades de ejercicio.

\section{ABSTRACT}

Spinning is a modality of aerobic activity. Their practice presents different choreographies, particularly in the variation of exercise intensity, which produce a wide spectrum in the responses of corporal metabolic variables, as the glucose. Their popularity and scarce information on the metabolic responses, we make the present study directed to value the glycaemia responses in a typical class of 1 hour duration and lipids levels in blood. Twenty-three overweight volunteers participated (IMC > $25 \mathrm{~kg} / \mathrm{m}^{2}$ ), (11 men and 12 women), average of age of $35 \pm 7$ years, without modifications in their habitual diet in previous week. The blood lipids went superior to the ideal values, and there was an increment in the lipid level, associated to hemoconcentration (6\%). The glucose increase $20 \mathrm{mg} / \mathrm{dL}$ and $14 \mathrm{mg} / \mathrm{dL}$ after exercise, in both men and women. Our data indicate security in the metabolic responses to exercise, when the exercise is practiced with method, graduating the intensity and the exercise intervals. More investigations will characterize this answer with other routines and exercise modalities.
L a práctica de spinning es una modalidad de actividad aeróbica, en la que los participantes son guiados por un instructor, mediante la cual se reproduce una secuencia de movimientos parecidos a una actividad de ciclismo de ruta. Las sesiones de entrenamiento

\section{INTRODUCCIÓN}

duran alrededor de una hora. La secuencia de actividades incluye: cinco a 10 minutos de calentamiento, 30 a 50 minutos de recorrido y terminan con cinco a 10 minutos de enfriamiento, en los cuales se incorporan ejercicios de estiramiento. La intensidad del ejercicio se regula por la actividad de la frecuencia cardiaca. Aunque su práctica es predominantemente
Citar como: Rivera-Cisneros AE, Sánchez-González JM, Cárdenas-González J, Noriega-Muro I, Martínez-Vega K, Gómez-Ballesteros FH et al. Práctica de spinning y respuesta metabólica en niveles de glucosa y lípidos séricos. Rev Mex Patol Clin Med Lab. 2020; 67 (3): 150-154. https://dx.doi.org/10.35366/96678 
Análisis Clínicos S.A, Chihuahua, Chih.

$\S \S$ Director del Laboratorio NIDIAC, S.A, Durango, Dgo.

Correspondencia: Dr. Antonio Eugenio Rivera-Cisneros E-mail: antonio.rivera. academico@gmail. com

Recibido: 29/09/2020 Aceptado: 20/10/2020 aeróbica, las coreografías pueden variar y ser de naturaleza anaeróbica o mixta (aeróbica y anaeróbica). ${ }^{1}$

Para que este ejercicio cumpla con los requisitos de ser benéfico para la salud o la enfermedad, se requiere efectuar actividades que no excedan $80 \%$ de la frecuencia cardiaca máxima (FC máx), que no existan variaciones importantes en los cambios de intensidad (idealmente no más de cinco a 10 latidos de diferencia) y a una frecuencia constante en el pedaleo (entre 80 y 100 revoluciones por minuto, RPM).

Esta actividad debe ser de naturaleza aeróbica para obtener los beneficios fisiológicos que se buscan en un paciente con enfermedad metabólica como el síndrome metabólico, la resistencia a la insulina, la diabetes o la obesidad, entre otras. Su práctica mejora el perfil de lípidos. Reduce $6 \%$ del colesterol total (CT), 10\% las lipoproteínas de baja densidad (LDLC), aumenta 5\% las lipoproteínas de alta densidad (HDLc), mejora el índice aterogénico $13 \%$ (CT/HDLc). ${ }^{2}$

No obstante, la hipoglucemia puede presentarse en pacientes con diabetes mellitus (DM), especialmente en los portadores de diabetes mellitus tipo 1 (DM T1), ya sea durante o después del ejercicio físico vigoroso. La hipoglucemia se presente entre una a 36 horas después de la práctica de ejercicio. La revisión de los niveles de glucosa, por tanto, deben comprenderse para evitar el riesgo en su práctica. ${ }^{3}$

Con el propósito de caracterizar la respuesta de la glucosa al spinning en pacientes con sobrepeso, se efectuó este estudio de tipo observacional, prospectivo, comparativo y transversal.

\section{MATERIAL Y MÉTODOS}

Participaron 23 voluntarios ( 11 hombres y 12 mujeres) con promedio de edad de $35 \pm 7$ años, quienes lo practicaron al menos dos veces a la semana en el año pasado, y que no tenían un régimen de alimentos controlado. Previa historia clínica, dirigida a identificar estados de comorbilidad y que pudieran afectar los niveles de glucosa o lípidos, los participantes fueron evaluados antropométricamente con peso, estatura y pliegues cutáneos; además, fueron derivados el índice de masa corporal y el contenido de grasa corporal. ${ }^{4,5}$

La semana previa al estudio, los voluntarios fueron instruidos para que la ingesta de sus alimentos se ajustara en las proporciones siguientes: hidratos de carbono 50 a $60 \%$; proteínas 12 a $22 \%$; grasas 25 a $35 \%$; colesterol $<200$ $\mathrm{mg} /$ día; fibra $\geq 30 \mathrm{~g} /$ día. ${ }^{6}$ El balance entre la ingesta y el gasto de energía diario no superó los $\pm 300 \mathrm{kcal} /$ día en ambos grupos.

Los estudios se efectuaron en el salón de un gimnasio, acondicionado para la práctica de spinning. Antes de iniciar una clase, a los pacientes se les efectuó venopunción en vena cefálica o basílica de manera estandarizada (reposo de cinco minutos previos a la punción, ayuno mínimo de ocho horas y máximo de 10 previo a la punción; posición del paciente sentado), para la extracción de muestras hemáticas, las que se colocaron en los tubos adecuados para su conservación y transporte al laboratorio, lo que se realizó en un lapso no mayor a 30 minutos una vez tomadas las muestras. Una vez coagulada la sangre, se realizó separación del paquete globular del suero y éste se mantuvo en refrigeración de cuatro a ocho grados hasta su proceso, dentro de las primeras tres horas.

La sesión tuvo una duración total de 60 minutos, la cual empezó con un calentamiento de 10 minutos, 40 minutos de actividad aeróbica entre 65 y $85 \%$ de la frecuencia máxima esperada para su edad (Karvonen; FC máxima esperada 220-edad), la cual fue monitoreada con un dispositivo electrónico comercial.

Cada uno de los sujetos otorgó por escrito su consentimiento de participación voluntaria, después de que les fueron explicados a cada uno los procedimientos, los riesgos y los beneficios del estudio, de acuerdo con lo establecido en la declaración de Helsinki. ${ }^{6-9}$

Una vez concluida la sesión, se procedió a tomar una nueva muestra de sangre (empleando el procedimiento estandarizado señalado previamente y manejando las muestras de la misma forma). La fórmula roja fue efectuada en contador celular automatizado Sismex K-1000, mientras que la glucosa, el colesterol total (CT) y triglicéridos (TG), por el método de química seca en equipo Vitros Slide. El colesterol de alta densidad (HDLc) y colesterol de baja densidad (LDLC) se efectuaron por técnica enzimática por 
espectrofotometría en un autoanalizador Alcyon de Abbott; las lipoproteínas de muy baja densidad (VLDLc) se obtuvieron por cálculo derivado, siguiendo para ello las indicaciones del fabricante del equipo. Los estudios estuvieron sujetos a un programa de control de calidad interno estandarizado, a fin de asegurar la validación de los resultados. Todo el proceso fue soportado también por un programa externo de la calidad, lo que, entre ambos programas, pudo garantizar la precisión y exactitud de las pruebas, con un coeficiente de variación intraensayo dentro del estándar.

El tamaño de la muestra se obtuvo a partir de la varianza esperada de los cambios en la glucosa hemática, de acuerdo con estudios previos efectuados por nuestro grupo. Se aplicó t pareada para determinar los cambios en las concentraciones de glucosa y las demás variables. El nivel de significancia estadística se fijó a un alfa de 95\%. Los datos fueron procesados en Statistica.

\section{RESULTADOS}

Siete de cada 10 participantes presentaron antecedentes familiares de algún tipo de enfermedad cronicodegenerativa como diabetes, enfermedad cardiovascular, hipertensión arterial, obesidad o dislipidemia. El contenido de grasa corporal excedió $25 \%$ en hombres y $28 \%$ en mujeres por antropometría. El contenido de la dieta fue positivo en su balance calórico. Presentaron un excedente en el consumo de calorías de 200 Kcal los hombres y 230 Kcal las mujeres.

Los hombres presentaron valores de frecuencia cardiaca (FC) en reposo y FC máx (181 \pm 10.7 versus $185.7 \pm$ 7.4 latidos por minuto) menores que las mujeres. Mientras que los valores de presión arterial sistólica, diastólica y media fueron mayores en hombres que en mujeres. El promedio de volumen máximo de oxígeno consumido o consumo máximo de oxígeno ( $\mathrm{VO}_{2}$ máx) en hombres fue $43.7 \mathrm{~mL} / \mathrm{kg} /$ minuto, con rango de 40.2 a 49.7 ; mientras que en mujeres de $38.2 \mathrm{~mL} / \mathrm{kg} /$ minuto, con rango de 30.4 a 47.6, de acuerdo con una prueba de Bruce, efectuada previamente sobre banda sin fin. Al correlacionar las características físicas de los participantes y su $\mathrm{VO}_{2}$ máx, se apreció relación inversa estadísticamente significativa, y entre porcentaje de grasa corporal y $\mathrm{VO}_{2}$ máx, tanto en el total de la muestra como en cada sexo.

Los valores hemáticos se muestran en la Tabla 1. El análisis de correlación entre el acondicionamiento físico y las concentraciones de lípidos séricos en reposo mostraron una moderada relación inversa entre $\mathrm{VO}_{2}$ máximo observado y la concentración de triglicéridos séricos en mujeres ( $r=-0.63, p=0.03$, no así en hombres. Durante la fase de calentamiento, el promedio de porcentaje de FC máxima fue ligeramente mayor en hombres que en mujeres ( $69 \pm 4.0$ versus $66 \pm 6.0 \%$ ). Durante los 40 minutos del recorrido, el promedio del porcentaje de FC máxima fue similar en ambos sexos (hombres $77 \pm$ $3.8 \%$, mujeres $76 \pm 2.4 \%$ ). En el enfriamiento también, el promedio del porcentaje FC máxima fue mayor en hombres ( $61 \pm 5.1$ versus $58 \pm 4.8 \%$ ).

Los hombres presentaron una mayor variación de la glucemia antes y después del ejercicio (20 vs $12 \mathrm{mg} / \mathrm{dL}$ ).

Se observó relación estadísticamente significativa entre $\mathrm{VO}_{2}$ máx y el porcentaje de variación de la glucosa, en el total de la muestra $(r=0.44, p=0.046)$, así como entre los hombres y las mujeres (Figura 1).

Las concentraciones de lípidos séricos después de la maniobra de estudio revelaron cambios significativos $(p<0.05)$ en todas las variables, tanto en hombres como en mujeres.

Los valores de hemoglobina y hematocrito se incrementaron como era esperado, por el fenómeno de

Tabla 1: Valores hemáticos encontrados antes y después de la prueba en hombres y mujeres $(\mathrm{N}=23)$.

\begin{tabular}{|c|c|c|c|c|}
\hline \multirow[t]{2}{*}{ Variable } & \multicolumn{2}{|c|}{ Hombres $(n=11)$} & \multicolumn{2}{|c|}{ Mujeres $(n=12)$} \\
\hline & Antes & Después & Antes & Después \\
\hline Hemoglobina (g/L) & $16.7 \pm 1.0$ & $17.8 \pm 0.7$ & $15.2 \pm 1.1$ & $16.6 \pm 1.3$ \\
\hline Hematocrito (\%) & $46.0 \pm 3.0$ & $48.0 \pm 1.3$ & $42.3 \pm 2.3$ & $44.6 \pm 2.6$ \\
\hline Proteínas (g/L) & $7.4 \pm 0.2$ & $8.1 \pm 0.1$ & $7.2 \pm 0.1$ & $7.8 \pm 0.1$ \\
\hline Glucosa (mg/dL) & $84.0 \pm 4.8$ & $104.5 \pm 8.3$ & $83.4 \pm 2.4$ & $95.6 \pm 1.7$ \\
\hline Colesterol total (mg/dL) & $210.0 \pm 22.3$ & $218.3 \pm 25.3$ & $194.6 \pm 28.9$ & $198.6 \pm 30.3$ \\
\hline Triglicéridos (mg/dL) & $155.0 \pm 57.0$ & $123.4 \pm 53.9$ & $117.4 \pm 46.4$ & $103.3 \pm 43.9$ \\
\hline $\mathrm{HDLC}(\mathrm{mg} / \mathrm{dL})$ & $34.7 \pm 3.9$ & $39.6 \pm 4.4$ & $53.4 \pm 2.4$ & $59.8 \pm 3.8$ \\
\hline LDLc (mg/dL) & $139.6 \pm 17.1$ & $146.3 \pm 19.8$ & $134.6 \pm 28.0$ & $139.1 \pm 27.5$ \\
\hline VLDLc (mg/dL) & $31.0 \pm 10.2$ & $24.6 \pm 10.1$ & $23.4 \pm 9.0$ & $20.6 \pm 9.1$ \\
\hline
\end{tabular}




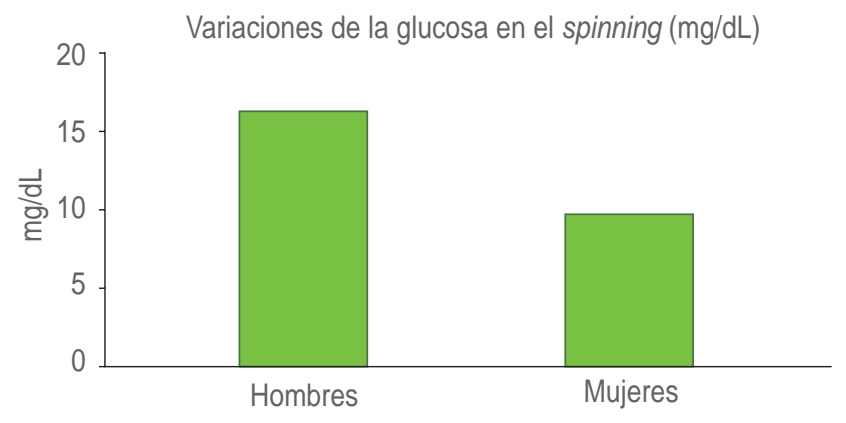

Figura 1: Variaciones de la glucemia en la prueba en hombres y mujeres $(\mathrm{N}=23)$.

hemoconcentración que se presenta en el ejercicio físico intenso y de larga duración.

\section{DISCUSIÓN}

Blair y su grupo indicaron que una dieta desequilibrada en el contenido energético y en la proporción de macronutrimentos se encuentra asociado a una corporalidad inadecuada a expensas de grasa corporal. ${ }^{10-12}$ La dieta de los participantes fue más alta en contenido energético y una importante desproporción en la ingesta de macronutrimentos, como el consumo de glucosa con base en alimentos refinados y lípidos séricos superior a los 60 y $30 \%$, respectivamente, que son las proporciones recomendadas para su ingesta diaria. El balance energético promedio fue positivo en ambos sexos. El consumo de fibra fue menor de $50 \%$ del recomendado. Los datos son consistentes con los reportados previamente. ${ }^{13}$

Por su parte, Brown y colaboradores ${ }^{14}$ indicaron que valores elevados de colesterol sérico total se presentan en individuos con índice de masa corporal superior a 25 $\mathrm{kg} / \mathrm{m}^{2}$. En nuestro estudio, los participantes presentaron valores no ideales para la salud humana, a pesar de tener niveles aceptables de capacidad cardiorrespiratoria (44.1 \pm 2.2 y $38.3 \pm 3.0$, para hombres y mujeres, respectivamente). Este hecho debe ser enfatizado en los programas de seguimiento a practicantes de la actividad física, para brindar consejo médico y nutricional, a efectos de contar con elementos para una práctica segura y efectiva de ejercicio físico. Giada ${ }^{5,6}$ concluyó que poseer un alto nivel de eficiencia aeróbica no es condición suficiente para modificar significativamente el perfil de lipoproteínas, lo cual coincide con lo reportado por Sánchez y equipo, ${ }^{13}$ quienes no encontraron relación entre el nivel de acondicionamiento físico y las concentraciones de lípidos séricos. Esta línea de pensamiento debe derribar la idea de que tener niveles elevados de aptitud física (en términos de $\mathrm{VO}_{2}$ máx) no garantiza tener salud cardiovascular. Por ello, el practicante de actividad física o deportista debe adoptar estilos de vida saludables concomitantemente a un programa de ejercicio físico metodológicamente adecuado.

La variación de la glucosa $(\Delta)$ fue de $20 \mathrm{mg} / \mathrm{dL}$ en los hombres y $14 \mathrm{mg} / \mathrm{dL}$ en las mujeres (Figura 1). El aumento de la glucosa como respuesta al ejercicio físico graduado es esperado, debido al incremento en los niveles de las hormonas reguladoras y contrarreguladoras de la glucosa, como es el caso de las catecolaminas, glucagón, cortisol y hormonas del crecimiento, las cuales se elevan como una respuesta al estrés causado por el ejercicio. ${ }^{7,15,16} \mathrm{La}$ magnitud del incremento de la glucosa es consistente con lo reportado por otros autores, ${ }^{17,18}$ quienes advierten en el cuidado que debe tenerse en personas portadoras de patologías como la diabetes mellitus tipo 1. Estos datos permiten apoyar la recomendación de que el spinning no altera significativamente las variaciones esperadas por la actividad aeróbica, cuando su práctica es realizada con metodología y procesos apropiados.

Los valores de hemoglobina y hematocrito reportados en nuestro estudio, se utilizaron para valorar el nivel o grado de hemoconcentración producido por la práctica de esta modalidad del ciclismo, y que potencialmente puede afectar los valores de las variables metabólicas estudiadas. La hemoconcentración, efecto de la pérdida de líquidos corporales debido al ejercicio, puede producir cambios en las concentraciones de lípidos séricos y prácticamente escasos en los niveles de glucosa, ya que por su peso molecular se difunde libremente. La variación en el volumen plasmático fue de $6 \%$, lo que se considera que no afectó a la respuesta de la glucosa.9,19

Los resultados sugieren la conveniencia de la práctica de esta modalidad de ejercicio físico, en especial por personas con riesgo de enfermedad cardiovascular. Sin embargo, es necesario ampliar la investigación en este tema, caracterizando distintas coreografías y produciendo variaciones en la intensidad del ejercicio, para establecer los límites en su práctica, particularmente en personas con sobrepeso, resistencia a la insulina o diabetes mellitus.

\section{REFERENCIAS}

1. Goldberg J. Manual del instructor de spinning. 5a edición; 1995.

2. Shephard RJ, Balady GJ. Exercise as cardiovascular therapy. Circulation 1999; 99:963-972.

3. Iscoe KE, Campbell JE, Jamnik V, Perkins BA, Riddell MC. Efficacy of continuous real-time blood glucose monitoring during and after prolonged high-intensity cycling exercise: spinning with a continuous glucose monitoring system. Diabetes Technol Ther. 2006; 8 (6): 627-635.

4. Fletcher GF, Balady GJ, Amsterdam EA, Chaitman B, Eckel R, Fleg $J$ et al. Exercise standards for testing and training: a statement for healthcare professionals from the American Heart Association. Circulation. 2001; 104: 1694-1740. 
5. Hellerstien HK, Franklin BA. ACSM's guidelines for exercise testing and prescription. Philadelphia: Lippincott Williams \& Wilkins; 2000.

6. USDA Dietary Guidelines for Americans 2005. Available in: www. healthierus.gov/dietaryguidelines.

7. Hornsby WG, Chetlin RD. Management of competitive athletes. Diabetes Spectr. 2005; 18: 102-107.

8. Asociación Médica Mundial. Declaración de Helsinki. Principios éticos para las investigaciones médicas en seres humanos enmendada en la 52.a Asamblea General. Edimburgo, Escocia: Asociación Médica Mundial; 2000.

9. Díaz-Ríos LK, Rivera-Cisneros AE, Macías-Cervantes MH, SánchezGonzález JM, Guerrero-Martínez FJ. Acute responses on lipid profile by practicing cycling. Rev Med Inst Mex Seguro Soc. 2008; 46 (2): 119-128.

10. Coyle EF. Physical activity as a metabolic stressor. Am J Clin Nutr. 2000; 72 (Suppl): 512S-520S.

11. Pollock M, Bohannon R, Cooper K. A comparative analysis of four protocols for maximal treadmill stress testing. Am Heart J. 1976; 92: 39-46.

12. Blair SN, Horton E, Leon AS, Lee IM, Drinkwater BL, Dishman RK et al. Physical activity, nutrition, and chronic disease. Med Sci Sports Exerc. 1996; 28: 335-349.
13. Reynaga-Ornelas ML, Rivera-Cisneros $A E$, Habacuc MM, Sánchez-González JM. Interacciones de la corporalidad: alimentación, lípidos séricos y máxima capacidad aeróbica en ejercitantes recreacionales. Rev Mex Patol Clin 1996; 43 (1): 27-34.

14. Brown CD, Higgins M, Donato KA, Rohde FC, Garrison R, Obarzanek E et al. Body mass index and the prevalence of hypertension and dyslipidemia. Obes Res. 2000; 8: 605-619.

15. Cauza E, Hanusch-Enserer U, Strasser B, Ludvik B, Kostner K, Dunky $A$ et al. Continuous glucose monitoring in diabetic long distance runners. Int J Sports Med. 2005; 26: 774-780.

16. Briscoe VJ, Tate DB, Davis SN, Type 1 diabetes: exercise and hypoglycemia. Appl Physiol Nutr Metab. 2007; 32: 576-582.

17. Herrington SJ, Gee DL, Dow SD, Monosky KA, Davis E, Pritchet $\mathrm{KL}$. Comparison of glucose monitoring methods during steady-state exercise in women. Nutrients. 2012; 4: 1282-1292.

18. Chassin LJ, Wilinska ME, Hovorka R. Intense exercise in type 1 diabetes: exploring the role of continuous glucose monitoring. J Diabetes Sci Technol. 2007; 1: 570-573.

19. Dill DB, Costill DL. Calculation of percentage changes in volumes of blood, plasma and red cells in dehydration. J Appl Physiol. 1974; 37 (2): $247-248$. 